\title{
A Comparison Between Coupled and Decoupled Vehicle Motion Controllers Based on Prediction Models*
}

\author{
Jose A. Matute ${ }^{1,2}$ and Ray Lattarulo ${ }^{1,2}$ and Asier Zubizarreta ${ }^{2}$ and Joshue Perez ${ }^{1}$
}

\begin{abstract}
In this work, a comparative study is carried out with two different predictive controllers that consider the longitudinal jerk and steering rate change as additional parameters, as additional parameters, so that comfort constraints can be included. Furthermore, the approaches are designed so that the effect of longitudinal and lateral motion control coupling can be analyzed. This way, the first controller is a longitudinal and lateral coupled MPC approach based on a kinematic model of the vehicle, while the second is a decoupled strategy based on a triple integrator model based on MPC for the longitudinal control and a double proportional curvature control for the lateral motion control. The control architecture and motion planning are exhaustively explained. The comparative study is carried out using a test vehicle, whose dynamics and low-level controllers have been simulated using the realistic simulation environment Dynacar. The performed tests demonstrate the effectiveness of both approaches in speeds higher than $30 \mathrm{~km} / \mathrm{h}$, and demonstrate that the coupled strategy provides better performance than the decoupled one. The relevance of this work relies in the contribution of vehicle motion controllers considering the comfort and its advantage over decoupled alternatives for future implementation in real vehicles.
\end{abstract}

\section{INTRODUCTION}

The vehicle driving tasks conventionally designed for human drivers are exponentially changing towards automation, giving the system the ability and authority to make decisions independently and self-sufficiently[1]. Several research and development efforts are heading to assure the safe execution of motion planning maneuvers under different traffic situations through virtual environments before to validate the dynamic behavior of the vehicle[2].

The ability of a vehicle system to successfully perform a dynamic driving task relies among other functions in the lateral and longitudinal vehicle motion control, including the detection of the vehicle position relative to the path and maintaining a settled speed[1]. In the literature, different control approaches have been proposed to implement lateral and longitudinal motion controllers, such as adaptive PID[3], Lyapunov-based approach[4], Model Reference Adaptive Controller (MRAC)[5] and several different focuses from vehicle control comparatives[6]. However, most of the approaches only focus on reference tracking, without

\footnotetext{
*This work has been conducted within the ENABLE-S3 project that has received funding from the ECSEL Joint Undertaking under Grant Agreement No 692455. This work was developed at Tecnalia Research \& Innovation facilities supporting this research.

${ }^{1}$ Industry and Transport Division, Tecnalia Research \& Innovation, San Sebastian, Spain joseangel.matute, ray.lattarulo, joshue.perez@tecnalia.com

${ }^{2}$ Automatic Control and System Engineering Department, University of the Basque Country, Bilbao, Spain asier.zubizarreta@ehu.eus
}

considering the comfort of the passengers. Moreover, there is a tendency to decouple lateral and longitudinal motion controllers, which could reduce the tracking performance. In contrast with previous strategies, the Model Predictive Control (MPC) permits to constraint physical parameters assuring safety and under a desired level of comfort[7].

Being a complex control problem, two strategies have been proposed when implementing the motion controllers. First, a coupled strategy in which the lateral and longitudinal control are considered in a single strategy [8], [9], which implies a more complex control problem. Second, a decoupled strategy, which allows to simplify the control problem by designing a controller for each task: one maintaining the lateral position of the vehicle along the road, and other following a speed reference [10], [11], [12], [13], [14].

Most of the previous controllers have the drawback that do not consider the comfort as a priority. Also, exists a tendency to decouple the lateral and longitudinal motion control, which means a simplification of the problem but reducing the performance and feeling comfort.

This way, in this work a comparative study is carried out considering two predictive controllers that consider comfort constraints in their formulations: a coupled lateral and longitudinal motion controller based on a modification of the kinematic bicycle model MPC proposed in[8]; and a decoupled approach that combines a triple-integrator model based MPC [7] for the lateral control combined with a double proportional curvature control for the lateral control[12]. The performance of both controllers and the coupling effect is analyzed considering a set of simulations using a realistic simulation environment, in which both the test vehicle and its low-level control have been modelled.

The rest of the work is organized as follows: SectionII develops each stage related with the control architecture developed for the vehicle motion control, detailing both proposed controllers. SectionIII details the simulation environment and the results of the tests performed, including the comparative analysis carried out. Finally, in SectionIV the most important ideas are summarized.

\section{Control Architecture}

This section describes the control architecture used in this investigation (Fig. 1). It begins with a global planner which contains all the path information for the vehicle. The local planner calculates how far is the vehicle from the desired path and if it is well oriented. The references provided by the local planner are to be executed by a control stage. In this work, two different MPC-based strategies are proposed, in 


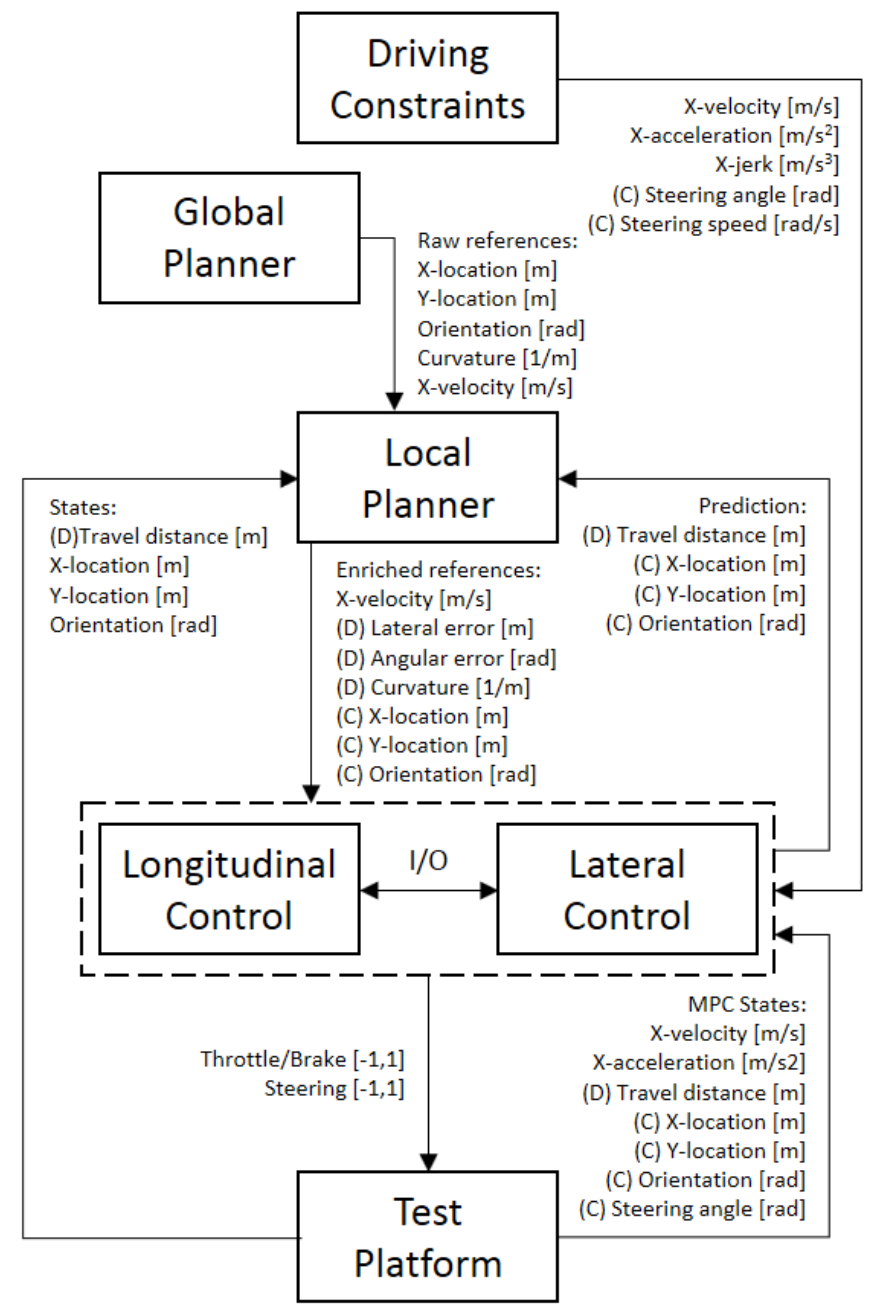

Fig. 1. Architecture for coupled and decoupled control

which the longitudinal and lateral vehicle motion control is presented in coupled and decoupled form. To execute these controllers some driving constraints as comfort are included. Finally, the test platform and the simulation environment area are presented.

\section{A. Global planner}

The designed route optimizes a space slightly smaller than 20 per 100 meters commonly used for real tests. An even number of maneuvers turning both to right and left attempt to reproduce real urban scenarios as roundabouts (RA), lane changes (LC) and intersections (INT). Smooth trajectories are obtained applying a parametric Bezier curve procedure [15]. To generate the route are necessary the entrance, exit and radius (for roundabouts) locations, and the design parameters (D). Each section of the route is designed with an even number of points $(\mathrm{N})$ of 21 .

An approximating polynomial $\gamma(T)=(X(T), Y(T))$ with $T \epsilon[-1,1]$ as a design variable is used to calculate the curvature $k$ based on derivatives [16] at each point of the route using the Eq. 1:

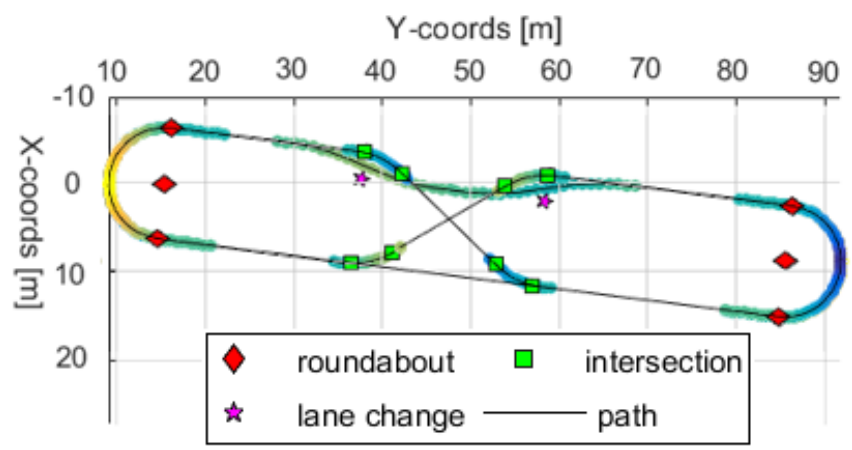

Fig. 2. Smoothed path with Bezier curves

$$
k=\frac{\left|X^{\prime} Y^{\prime \prime}-Y^{\prime} X^{\prime \prime}\right|}{\left(X^{\prime 2}+Y^{\prime 2}\right)^{\frac{3}{2}}}
$$

where $X$ and $Y$ are the coordinates expressed in the global reference frame depicted in Fig. 4.

The reference velocity $v_{r e f}$ along the desired route is calculated using Eq. 2. A comfort criteria is introduced considering a permissible total acceleration to be felt by the passengers $a_{w}$ [17]. $k$ is the curvature of each point in the route. The weight index $\left(n_{w}=1.4\right)$ is based on the standard ISO2631-1.

$$
v_{r e f}=\sqrt{\frac{a_{w}}{n_{w} k}}
$$

The route in local coordinates and orientations, as well as the main points for the different maneuvers planned using Bezier curves are shown in Fig. 2. The curvature and velocities obtained from Eqs. 1-2.

\section{B. Local Planner}

In this stage the differences between the location of the vehicle and the route from the Global Planner are calculated according with the parameters depicted in Fig. 4. As the route is divided in consecutive straight segments, if the projection of the vehicle onto the segment is out of bounds the previous or the next segment must be selected to make a new projection. Eq. 3 is useful to know if the projection of the vehicle is on the segment:

$$
v=\frac{\left(X_{i}-X_{0}\right)\left(X_{1}-X_{0}\right)+\left(Y_{i}-Y_{0}\right)\left(Y_{1}-Y_{0}\right)}{\left(X_{1}-X_{0}\right)^{2}+\left(Y_{1}-Y_{0}\right)^{2}}
$$

where the value of $v$ must be from 0 to 1 for a correct projection of the vehicle on the segment of the route. A previous or next segment will be selected in case of the value of $v$ be negative or higher than 1 , respectively. This means that the projection is out of bounds.

After the projection of the vehicle is onto the selected segment, the perpendicular distance of the vehicle to the route and the difference in their orientations are calculated, being these the lateral and angular errors. A flow diagram of this process is shown in Fig. 3. 
Parameters from the route planner as $v_{x}, X, Y, \Psi$ (Fig. 4) and the curvature $k$ are taken from the selected segment of the route as references for the control. Eqs. 4 and 5 show the calculation of vehicle's lateral and angular errors with respect to a route segment.

$$
\begin{gathered}
e_{y}=\frac{\left(Y_{i}-Y_{0}\right)\left(X_{1}-X_{0}\right)-\left(X_{i}-X_{0}\right)\left(Y_{1}-Y_{0}\right)}{\left(X_{1}-X_{0}\right)^{2}+\left(Y_{1}-Y_{0}\right)^{2}} \\
e_{\phi}=\arctan 2\left(\sin \left(\Psi_{i}-\Psi_{0}\right), \cos \left(\Psi_{i}-\Psi_{0}\right)\right)
\end{gathered}
$$

\section{Vehicle Motion Control}

In this section, the lateral and longitudinal control approaches are detailed. As stated in the introduction, two control approaches will be detailed and compared. The first is based on a coupled approach, that used a kinematic bicycle model to perform lateral and longitudinal MPC control. The second is a decoupled approach, in which a triple integrator model is used to implement a longitudinal MPC control, and a double proportional curvature control for the lateral control.

For the rest of the section, the following nomenclature is used: the $x$ and $y$ are the coordinates relative to the longitudinal and lateral axis of the vehicle with origin in the center of gravity, $X$ and $Y$ are the global coordinates, $\Psi$ is the heading angle of the vehicle, $d_{x}$ is the path travel distance, $v_{x}$ is the longitudinal speeds, $a_{x}$ is the longitudinal acceleration, $\delta$ is the front wheel steering angle, and the $j_{x}$ and $\Delta \delta$ are the longitudinal jerk and the front wheel steering rate change of the vehicle, respectively.

In order to detail the developed controllers, this section is divided as follows: In section II-C.1 the Kinematic Bicycle model used in the coupled controller is detailed. Section IIC. 2 details the triple integrator model used in the decoupled controller. Section II-C.3 specifies the MPC formulation. Section II-C.4 explains the considered driving constraints, which are applied to both controllers. Finally, Section IIC.5 mentions the toolbox employed to solve the MPC formulation.

1) Kinematic Bicycle Model for the Coupled Controller: The kinematic bicycle model is used in the MPC coupled controller to consider lateral and longitudinal coupled behavior of the vehicle. The two front and rear wheels are lumped into a unique wheel in the centers of the front and rear axles, respectively (Fig. 4). The constant $L$ denotes the wheelbase

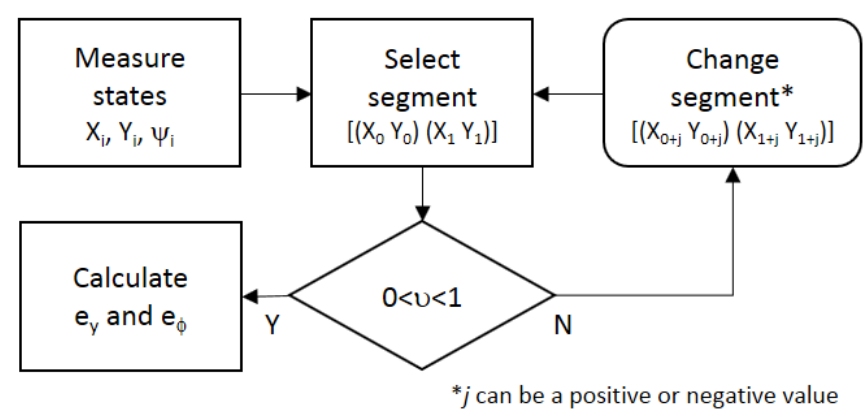

Fig. 3. Route tracker in control architecture

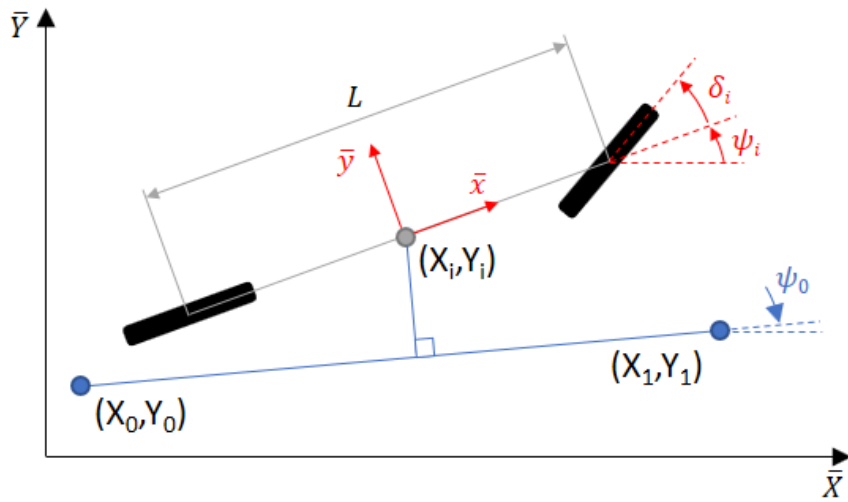

Fig. 4. Simplified kinematic bicycle model of the vehicle

of the vehicle. Focusing in comfort, this model differs from [8] in the addition of Eqs. $7 \mathrm{~b}$ and $7 \mathrm{f}$ permitting to choose the rate change of physical control devices. Also, the slip angle variable is removed from the original system of equations detailed in [18] due to its negative effect in the longitudinal vehicle motion control. Eqs. 7a-7f and the following explicit ODE formulation describes the kinematic bicycle model:

$$
\begin{array}{lll}
\dot{d}_{x}=v_{x} & \text { (6a) } & \dot{v}_{x}=a_{x} \\
\dot{v}_{x}=a_{x} & \text { (6b) } & \dot{a}_{x}=j_{x} \\
\dot{a}_{x}=j_{x} & \text { (6c) } & \dot{X}=v_{x} \cos (\Psi) \\
& & \dot{Y}=v_{x} \sin (\Psi) \\
& \\
& & \\
& & =v_{x} \tan (\delta) \\
L & \dot{\delta}=\Delta \delta
\end{array}
$$

2) Triple Integrator Model for the Decoupled Controller: A triple-integrator is implemented to model the longitudinal kinematic of the vehicle in the proposed decoupled controller[7]. In this approach, the vehicle is considered as an ideal particle which is considered as an ideal particle traveling along a predefined route. An explicit ODE formulation describing the longitudinal model of the vehicle is represented in Eqs. 6a-6c:

For the lateral control of the vehicle, the lateral and angular errors, and the curvature $k$ of the path are used as proposed in [12]. This strategy has proven to be useful in the tracking of routes with curvatures of variable ratio. The headway distance varies with the speed of the vehicle, taking a point that is $0.3 \mathrm{~s}$ in front of the center of gravity of the vehicle. Eq. 8 specifies the lateral control calculation:

$$
U_{y}(t)=G_{1} k+G_{2} e_{y}+G_{3} e_{\phi}
$$

where the constants $G_{1}, G_{2}$ and $G_{3}$ are the controller gains which values are selected as $1,0.1$ and -1 , respectively.

3) MPC Formulation: The linear and nonlinear vehicle dynamics described for a coupled strategy in Eqs. 7a-7f or a decoupled strategy in Eqs. 6a-6c can be expressed as the following general compact form: 


$$
\frac{d \chi}{d t}=f(\chi(t), u(t))
$$

where the state and control parameters are different depending of the model employed. For the triple-integrator model are $\chi=\left[\dot{d}_{x}, \dot{v_{x}}, \dot{a_{x}}\right]^{T}$ and $u=j_{x}$. For the kinematic bicycle model are $\chi=\left[v_{x}, \dot{a}_{x}, \dot{X}, \dot{Y}, \dot{\Psi}, \dot{\delta}\right]^{T}$ and $u=\left[j_{x}, \Delta \delta\right]^{T}$.

The aim of the control task consists to follow a defined trajectory under specific velocities from an initial position with zero speed in the track. The vehicle dynamics for a coupled or decoupled strategy has a fixed time step $T_{s}$. The output states are defined for the coupled control in Eq. 10b and for the decoupled control in Eq. 10a as follows:

$$
\begin{gathered}
\eta(k)=h(\chi(k))=\left[\begin{array}{llll}
0 & 1 & 0
\end{array}\right] \chi(k) \\
\eta(k)=h(\chi(k))=\left[\begin{array}{cccccc}
1 & 0 & 0 & 0 & 0 & 0 \\
0 & 0 & 1 & 0 & 0 & 0 \\
0 & 0 & 0 & 1 & 0 & 0 \\
0 & 0 & 0 & 0 & 1 & 0
\end{array}\right] \chi(k)
\end{gathered}
$$

The next cost function is considered for the optimization:

$$
J(\chi(t), u(t))=\sum_{i=1}^{H}\left\|\eta_{t+i, t}-\eta_{t+i, t}^{r e f}\right\|_{Q}^{2}+\left\|u_{t+i, t}\right\|_{R}^{2}
$$

where $\eta=v_{x}$ for the triple-integrator model and $\eta=$ $\left[v_{x}, x, y, \Psi\right]^{T}$ for the kinetic bicycle model with $\eta_{\text {ref }}$ as the respective reference parameter. The first summand denotes the penalty on speed reference and trajectory errors while the second one measures the command signals to the actuation devices. The weighting matrices $Q$ and $R$ are defined intuitively in order to provide a balance between safety and comfort as $Q=\operatorname{diag}([1,10])$ and $R=[1]$ for the triple integrator model, and $Q=\operatorname{diag}([1,1,1,1,1,1])$ and $R=\operatorname{diag}([1,1,1,1])$ for the kinetic bicycle model. The finite horizon optimal control problem formulation is solved at each step $t$ as:

$$
\begin{array}{r}
\min _{\eta(\cdot), u(\cdot)} J\left(\chi_{t}, u_{t}\right) \\
\text { s.t. } \quad \chi_{k+1, t}=f^{d t}\left(\chi_{k, t}, u_{k, t}\right) \\
\eta_{k, t}=h\left(\chi_{k, t}\right) \\
k=t, \ldots, t+H \\
\zeta_{f, \min } \leq \zeta_{k, t} \leq \zeta_{f, \max } \\
u_{f, \min } \leq u_{k, t} \leq u_{f, \max } \\
\chi_{t, t}=\chi(t)
\end{array}
$$

where $\eta_{t+i, t}$ is the output prediction at time $t+i$ beginning from state $\chi_{t, t}=\chi(t), H$ denotes the output prediction horizon. At the next step $t+1$, the optimal problem is solved again with a new horizon based on a new measurement of the states $\chi(t+1)$.
One of the improvements of the control stage proposed is the possibility to define the level of driving comfort through the definitions of longitudinal jerk and steering rate change as control constraints. The limitation of this parameters helps to reduce sudden changes in acceleration, braking and steering. The MPC outputs $j_{x}$ and $\Delta \delta$ are numerically integrated before the next stage to be used as control parameters.

4) Driving Constraints: The lower and upper bounds for the state $\left(\zeta_{k, t}\right)$ in Eqs. 13a, 13b and 13d, and control constraints $\left(u_{k, t}\right)$ in Eqs. 13c and 13e are selected according with the MPC model employed:

$$
\begin{array}{rr}
0 \leq v_{k, t}^{x} \leq v_{k, t}^{r e f} \\
-3 \leq a_{k, t}^{x} \leq 1 \\
-2 \leq j_{k, t}^{x} \leq 2 \\
\text { kinetic-bicycle } & -0.52 \leq \delta_{k, t} \leq 0.52 \\
& -0.50 \leq \Delta \delta_{k, t} \leq 0.50
\end{array}
$$

where the constraints defined for the triple-integrator model are also applied to the kinetic bicycle model at each iteration $t$. The route planner of Section II-A specifies the references $\eta_{k, t}$ for the entire horizon.

5) MPC Solver: The open-source ACADO Toolkit is used to solve the optimal control problem previously described [19]. The time continuous Optimal Control Problem (OCP) is reformulated to an approximate nonlinear program (NLP) using the direct multiple shooting discretization method. The generalized Gauss-Newton approximation iterates by solving the Sequential Quadratic Program (SQP) algorithm to solve the NLP. The SQP is then solved by the dense linear algebra solver qpOASES3. A continuous output Implicit RungeKutta of Gauss-Legendre integrator of order 2 is exported by the code to simulate the system with 20 integration steps. The horizon estimation is parametrized to obtain $H=10$ elements of $0.3 \mathrm{~s}$ each of uniform duration.

\section{Test Vehicle}

The comparative study proposed in this investigation is performed in Dynacar[20]. This is an environment and vehicle dynamics simulator integrated in the control architecture shown in Fig. 1 as a generated $\mathrm{C}$ code compiled in a MEX function running in MATLAB/Simulink 2015b. The simulation environment consists of a plain circuit that contains several traffic features, reproducing an ideal scenario generally used for real automated driving tests.

The vehicle model relies on a multi-body formulation of 14 degrees of freedom (DOF) [11]. The chassis and wheels are linked through knuckle-type suspensions relating their movements through look-up tables. This formulation design permits to apply independent torques on each wheel being broadly flexible in the integration of any powertrain or braking model.

The simulated vehicle selected for the simulated test platform is an electric Renault Twizy 80. The characteristics are depicted in Table I. The CG location is measured from the front axle of the vehicle. 
TABLE I

RENAULT TWIZY 80 TECHNICAL SPECIFICATIONS

\begin{tabular}{ccc}
\hline Mass & 611.50 & $\mathrm{~kg}$ \\
Dimensions & $2.34 \times 1.23 \times 1.45$ & $\mathrm{~m}$ \\
CG location & $-0.93 \times 0.00 \times 0.49$ & $\mathrm{~m}$ \\
Wheelbase & 1.69 & $\mathrm{~m}$ \\
Trackwidth & 1.09 & $\mathrm{~m}$ \\
Inertia & $243.18,430.17,430.17$ & $\mathrm{~kg}-\mathrm{m}^{2}$ \\
Front wheel radius & 0.27 & $\mathrm{~m}$ \\
Rear wheel radius & 0.28 & $\mathrm{~m}$ \\
Steering ratio & $14.27: 1$ & - \\
\hline Traction torque & 57 & $\mathrm{~N}-\mathrm{m}$ \\
Transmission ratio & $1: 9.23$ & - \\
Braking torque & 500 & $\mathrm{~N}-\mathrm{m}$ \\
\hline
\end{tabular}

The command signals for the throttle and brake pedals come from the control stage as normalized values to the test platform. Therefore, some manipulations must be done to transform them into torque values. The low-level control devices acting on the steering wheel, throttle and brake pedals are simplified and implemented as shown next.

1) Low Level Control Model: The devices involved in this stage of the control architecture are commonly associated with servomotors acting directly over mechanical components of the vehicle. Consequently, gains, delays and rate change limiters must be considered in the model to mimic the behavior of actuation devices in real applications.

A gain is associated with the amplitude of the command signals usually transformed after passing through intermediate devices as programming logic controllers (PLC) or Electric Control Units (ECU) installed in the vehicles. This variable is most of the times smaller than the original signal for the safety of mechanical and electrical systems.

Time delays are considered a critical issue in low level control, as the command signals are not executed instantly by the actuation devices. This parameter is mainly associated with the delays of the internal communications in the vehicle.

The time constant $(\tau)$ of the actuator models (usually first order systems) is also an important factor when designing the control system. This behavior in physical devices comes from the actuation speed that is not immediate. The stabilization of electrical signals also presents a similar behavior in lower order of magnitude.

A rate limiter is also a useful parameter to be considered in control systems to constraint the speed of change in the position of actuation devices. The values of the parameters implemented are depicted in the Table II.

2) Steering, Powertrain and Brake Models: The steering wheel angle rotation to one side has a maximum value of $8.80 \mathrm{rad}$ which is directly related to the front wheels through a steering ratio.

The motor torque is divided and delivered evenly to the

TABLE II

CHARACTERISTICS OF LOW LEVEL OF CONTROL

\begin{tabular}{|c|c|c|c|c|}
\hline & Gain & delay [ms] & $\tau[\mathbf{m s}]$ & limiter $\left[s^{-1}\right]$ \\
\hline Throttle & 0.57 & 50 & 200 & - \\
\hline Brake & 0.43 & 100 & 200 & - \\
\hline Steering & 0.71 & 50 & - & 0.50 \\
\hline
\end{tabular}

two rear wheels after being incremented and reoriented by the gearbox. A general mechanical efficiency in the driveline (90\%) is considered. Considering that simulation tests are always performed at speeds lower than $10 \mathrm{~m} / \mathrm{s}$ is assumed that the motor is always delivering a constant torque.

The hydraulic braking torque is divided and evenly applied in the four wheels. A delay in this kind of systems related with the lumped lag of the hydraulic system is considered.

\section{RESUltS AND DISCUSSION}

In this section, the results of a comparative study between the coupled and decoupled strategies of control defined in Section II-C. The test circuit defined Fig. (2) is suitable to replicate several traffic urban conditions under specific comfort parameters for passengers or goods transportation. The results are presented as function of the travel distance (R) on route.

In Fig. 5 the velocity reference, as well as the lateral and angular errors along the route are depicted. The lateral error presents a phase difference of nearly 180deg between the two control strategies. The coupled technique has a better prediction of the lateral changes which allows the vehicle to circulate in the inside part of the curves while turning in contrast with the decoupled approach which uses the outside of the curves.

The decoupled control becomes unstable at high speeds in several sectors of the route. The angular error of the coupled approach is slightly better and no important instability is observed. The reference velocity is correctly followed and predicted by both strategies reaching a max speed of $33 \mathrm{~km} / \mathrm{h}$.

In Table III a comparison between the longitudinal and lateral behavior in terms of peak-to-peak (p-p) and rootmean-square (rms) errors is presented. The coupled strategy shows a better performance in the route tracking both lateral and angular for each error calculation.

The whisker plots presented in the Fig. 6 shows the statistical data of the analysis of the lateral and angular errors results obtained from the simulations of the coupled (C) and decoupled (D) vehicle motion controllers. Although the median value of the decoupled controller $(0.04 \mathrm{~m})$ is lower

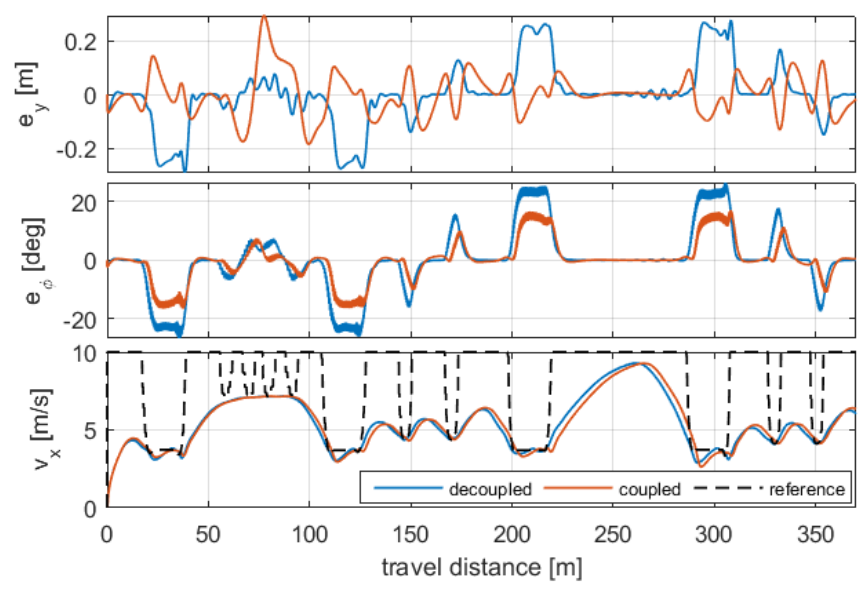

Fig. 5. Errors and speed reference 
TABLE III

LATERAL AND ANGULAR ERRORS

\begin{tabular}{cccc}
\hline measure & parameter & decoupled & coupled \\
\hline $\mathrm{p}-\mathrm{p}$ & $e_{y}[\mathrm{~m}]$ & 0,57 & 0,48 \\
& $e_{\Psi}[\mathrm{deg}]$ & 53,16 & 33,71 \\
\hline rms & $e_{y}[\mathrm{~m}]$ & 0,13 & 0,07 \\
& $e_{\Psi}[\mathrm{deg}]$ & 12,06 & 7,60
\end{tabular}

than the coupled one $(0.05 \mathrm{~m})$, the $50 \%$ of the central data is lower for the coupled controller $(0.06 \mathrm{~m})$ having a less dispersion of the data in comparison with the decoupled controller. A similar behavior regarding with the dispersion is found in the angular error results, the coupled controller shows the best performance.

\section{CONClusions AND Future Work}

The results of this work permit to evaluate two strategies for vehicle motion control commonly found in automated driving architectures. The model predictions obtained from the MPC are essential to modify the longitudinal velocity of the vehicle, satisfying the speed limit values defined in the Route Planner stage and therefore obtaining the desired level of lateral accelerations along the route.

The decoupled control strategy presents instabilities due mainly to high speeds. To avoid this problem is necessary to change the value of the lateral controller gains according with the range of velocity performed, being this value inherent to a single vehicle and being no possible to use the exact same values to control another vehicle of different characteristics of size and weight.

The coupled control strategy covers a larger range of velocities obtaining negligible instability responses. For the tests developed stable responses are obtained having important speed changes as from $4 \mathrm{~m} / \mathrm{s}$ to $10 \mathrm{~m} / \mathrm{s}$. This strategy uses the same parameter weights in the controller for all the range of velocities with no need of modifications to obtain good results. In comparison with the decoupled control, the rate change in the steering wheel control is considered, being this also an important factor to obtain smooth and stable results.

The best results are obtained using a coupled control strategy, having a complete prediction for the status of the vehicle in longitudinal and lateral parameters. This slows to optimize the status from references considering driving constraints accordingly.

Future works will considerate the employment of this strategies for real vehicle implementation, evaluating the response under the influence of real sensor, low level control and non-linearities in the test platform.

\section{REFERENCES}

[1] S. International, "Taxonomy and definitions for terms related to onroad motor vehicle automated driving systems j3016," in J3016, p. 35, 2018.

[2] P. Heidl and W. Damm, "Safetrans working group," in Highly automated Systems: Test, Safety, and Development Processes, p. 19, 2016.

[3] J. Monteil, J. Sau, and M. Bouroche, "Adaptive pid feedback control for the longitudinal dynamics of driver-assisted vehicles in mixed traffic," in 2016 IEEE 19th International Conference on Intelligent Transportation Systems (ITSC), pp. 1634-1641, Nov 2016.

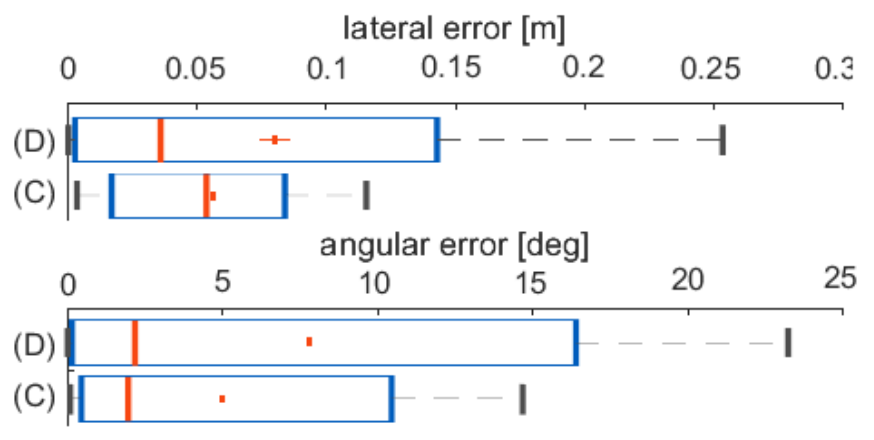

Fig. 6. lateral and angular errors

[4] R. Attia, R. Orjuela, and M. Basset, "Combined longitudinal and lateral control for automated vehicle guidance," Vehicle System Dynamics, vol. 52, no. 2, pp. 261-279, 2014.

[5] A. Raffin, M. Taragna, and M. Giorelli, "Adaptive longitudinal control of an autonomous vehicle with an approximate knowledge of its parameters," in 2017 11th International Workshop on Robot Motion and Control (RoMoCo), pp. 1-6, July 2017.

[6] D. Calzolari, B. Schrmann, and M. Althoff, "Comparison of trajectory tracking controllers for autonomous vehicles," in IEEE 20th International Conference on Intelligent Transportation Systems (ITSC), 2018.

[7] J. A. Matute, M. Marcano, A. Zubizarreta, and J. Perez, "Longitudinal model predictive control with comfortable speed planner," in 2018 IEEE International Conference on Autonomous Robot Systems and Competitions (ICARSC), pp. 60-64, April 2018.

[8] J. Kong, M. Pfeiffer, G. Schildbach, and F. Borrelli, "Kinematic and dynamic vehicle models for autonomous driving control design," in IEEE Intelligent Vehicles Symposium (IV), pp. 1094-1099, 2015.

[9] P. Polack, F. Altché, B. d'Andréa Novel, and A. de La Fortelle, "Guaranteeing consistency in a motion planning and control architecture using a kinematic bicycle model," arXiv, 2018.

[10] S. Dominguez, A. Ali, G. Garcia, and P. Martinet, "Comparison of lateral controllers for autonomous vehicle: Experimental results," in 2016 IEEE 19th International Conference on Intelligent Transportation Systems (ITSC), pp. 1418-1423, Nov 2016.

[11] M. Marcano, J. A. Matute, R. Lattarulo, E. Marti, and J. Perez, "Low speed longitudinal control algorithms for automated vehicles in simulation and real platforms," Complexity, p. 12 pages, 2018.

[12] D. Gonzalez and J. Perez, "Control architecture for cybernetic transportation systems in urban environments," in 2013 IEEE Intelligent Vehicles Symposium (IV), pp. 1119-1124, June 2013.

[13] R. Solea and U. Nunes, "Trajectory planning and sliding-mode control based trajectory-tracking for cybercars," Integrated Computer-Aided Engineering, vol. 14, pp. 33-47, 2007

[14] J. M. Snider et al., "Automatic steering methods for autonomous automobile path tracking," Robotics Institute, Pittsburgh, PA, Tech. Rep. CMU-RITR-09-08, 2009.

[15] R. Lattarulo, L. Gonzalez, E. Marti, J. Matute, M. Marcano, and J. Perez, "Urban motion planning framework based on n-bzier curves considering comfort and safety," Journal of Advanced Transportation, 2018.

[16] S. Hermann and R. Klette, "A comparative study on 2d curvature estimators," in 2007 International Conference on Computing: Theory and Applications (ICCTA'07), pp. 584-589, March 2007.

[17] D. Gonzlez, J. Prez, R. Lattarulo, V. Milans, and F. Nashashibi, "Continuous curvature planning with obstacle avoidance capabilities in urban scenarios," in 17th International IEEE Conference on Intelligent Transportation Systems (ITSC), pp. 1430-1435, Oct 2014.

[18] R. Rajamani, Vehicle Dynamics and Control. Mechanical Engineering Series, Springer US, 2011.

[19] R. Quirynen, M. Vukov, M. Zanon, and M. Diehl, "Autogenerating microsecond solvers for nonlinear mpc: A tutorial using acado integrators," Optimal Control Applications and Methods, vol. 36, November 2014.

[20] I. Iglesias-Aguinaga, A. Martin-Sandi, and A. Pea-Rodriguez, "Vehicle modelling for real time systems application. the virtual rolling chassis," DYNA, vol. 88, no. 2, pp. 206-215, 2013. 